\title{
Heriot-Watt SCHOOLS Physics Laboratory
}

\author{
Summary \\ A collaborative venture, set up by the Physics Department of Heriot-Watt University, \\ Edinburgh, to provide practical support for school Physics departments in carrying out \\ and assessing the practical elements of SQA Higher and Advanced Higher Physics. \\ The venture allows school students to work in a university environment and in this \\ way strengthens the links between school and university.
}

Subject area: Physics

\section{Description}

The work provides practical support for school Physics departments in carrying out and assessing the practical elements of the Scottish Qualifications Authority Higher and Advanced Higher Physics syllabuses and in so doing strengthens the links between school and university.

\section{Type of activity}

Laboratory work related to the SQA Higher and Advanced Higher Physics syllabuses.

\section{Content covered}

Practical experimental work for assessment and moderation in each of the three Units of the SQA Higher and Advanced Higher Physics Courses.

Practical experimental work for assessment and moderation for the Physics Investigations half-unit for the SQA Advanced Higher Physics Course.

\section{Application}

The SCHOOLS Physics Laboratory was set up in the Physics Department of HeriotWatt University to provide schools with practical help in carrying out the experimental aspects of both the Higher and the Advanced Higher Physics Courses. It does this by giving school students the opportunity to carry out experimental work in the Physics Department of the University, using prepared experimental worksheets or 'scripts'. These scripts have been written in such a way as to enable students to carry out the experimental work even before the relevant theory has been covered by the teacher in class.

The Higher Physics Course consists of three Units - 'Mechanics and Properties of Matter', Electricity and Electronics' and 'Radiation and Matter'. For each of these Units, students were required to perform practical work and submit a report of one experimental activity. (This requirement has subsequently been amended to only one report from any one of the three Units of the complete Course.) This requirement is known as the 'Learning Outcome 3' or 'LO3' report. A similar requirement exists for the $2 \frac{1}{2}$ theory Units of the Advanced Higher Physics Course. In addition, the Advanced Higher Course contains a half Unit (20 hours nominally) called 'Physics Investigation'. 
When the SCHOOLS Laboratory was first set up, in November 2000, the work carried out by students in the laboratory was aimed primarily at those experiments relating to the Advanced Higher Course that were difficult, if not impossible, for schools to carry out, and which were suitable for LO3 assessments. For example, the 'Cavendish-Boys determination of the gravitational constant $G$ ', requires apparatus not generally available in schools and is too time-consuming to carry out at school. The 'Coulomb's Law' apparatus is too delicate (and too expensive) to be set up in a school classroom that may have a junior class coming in immediately after its use by responsible seniors. For an accurate determination of the 'Force on a current-carrying conductor', a balance with a capability of measuring a small fraction of a gram is needed. Such a balance may well use up the complete funding allocation of a school Physics department for one year. In the SCHOOLS laboratory several of the experiments that have been set up are interfaced to dedicated computers. To tie up computers in this way is impossible in most school Physics departments.

The SCHOOLS Laboratory has since been extended in its scope, to include experimental work related to the content of the Higher Physics Course, although, in general, schools are more able to cope with these needs since the equipment requirements are not so demanding either in cost or complexity.

A more recent expansion of the SCHOOLS Laboratory has been into the area of Advanced Higher Physics Investigations. This has been in response to the many requests received from schools to provide some much-needed help with the Physics Investigations half-unit. One way of meeting the requirements of this half unit is for the school students to carry out a series of short experiments linked to each other, in a particular area of Physics. This is the approach that has been used in the SCHOOLS Laboratory. The students from a particular school will typically spend three days, perhaps in consecutive weeks, working on themed experiments. Some of the Investigations that have been successfully carried out include Polarisation of Light; The Wavelength of Light; Inverse Square Law Relationships; Gravity; Factors Affecting the Force on a Current Carrying Conductor; Sound Investigations; Rotational Dynamics.

In using the SCHOOLS Laboratory, there are many benefits to the school Physics department, to the school students individually, and to the Physics Department of Heriot-Watt University. The school Physics department benefits because the experiments that the school students carry out are all closely related to the theory work in the Higher or Advanced Higher Course. The experiments meet the criteria required by the SQA for the assessment of Learning Outcome 3 or the Physics Investigations Unit as appropriate, so reducing the assessment burden on school Physics departments. As already explained, the experiments are, in general, the ones that for various reasons, schools have difficulty in offering to school students.

The school students benefit because they are given the opportunity to carry out experiments that they may not be able to do otherwise. Since all of the experiments are either computer-interfaced or use computers for data-handling, students become familiar with a greater variety of equipment and computer hardware and software. The SCHOOLS Laboratory is housed in the first-year undergraduate laboratory in the Physics Department of Heriot-Watt, so school students work in a university teaching laboratory and can begin to experience university life at first hand. The school 
students can also take the opportunity to speak to members of the university staff as well as undergraduate and postgraduate students.

Heriot-Watt Physics Department benefits from the closer liaison with school Physics teachers and students in schools. The SCHOOLS Laboratory offers an opportunity for the University to 'open its doors', in a very practical way, to its possible future undergraduates.

Such has been the success of the SCHOOLS Laboratory, that geography has not proved to be a constraint. As well as schools in the immediate vicinity of the University making use of the facilities offered, there have been visits, and indeed return visits in subsequent years, from schools as far away as Islay and Fortrose on the Black Isle. Both of these visits involved an overnight stay.

One Physics teacher, on a return visit to use the SCHOOLS Laboratory, commented that the passes achieved by his Advanced Higher class of the previous year had been the best he had ever achieved in his teaching career. While this success could not be entirely attributed to the visit to the SCHOOLS Laboratory, he was of the opinion that the experience that has been offered to that class had been a contributory factor in their success.

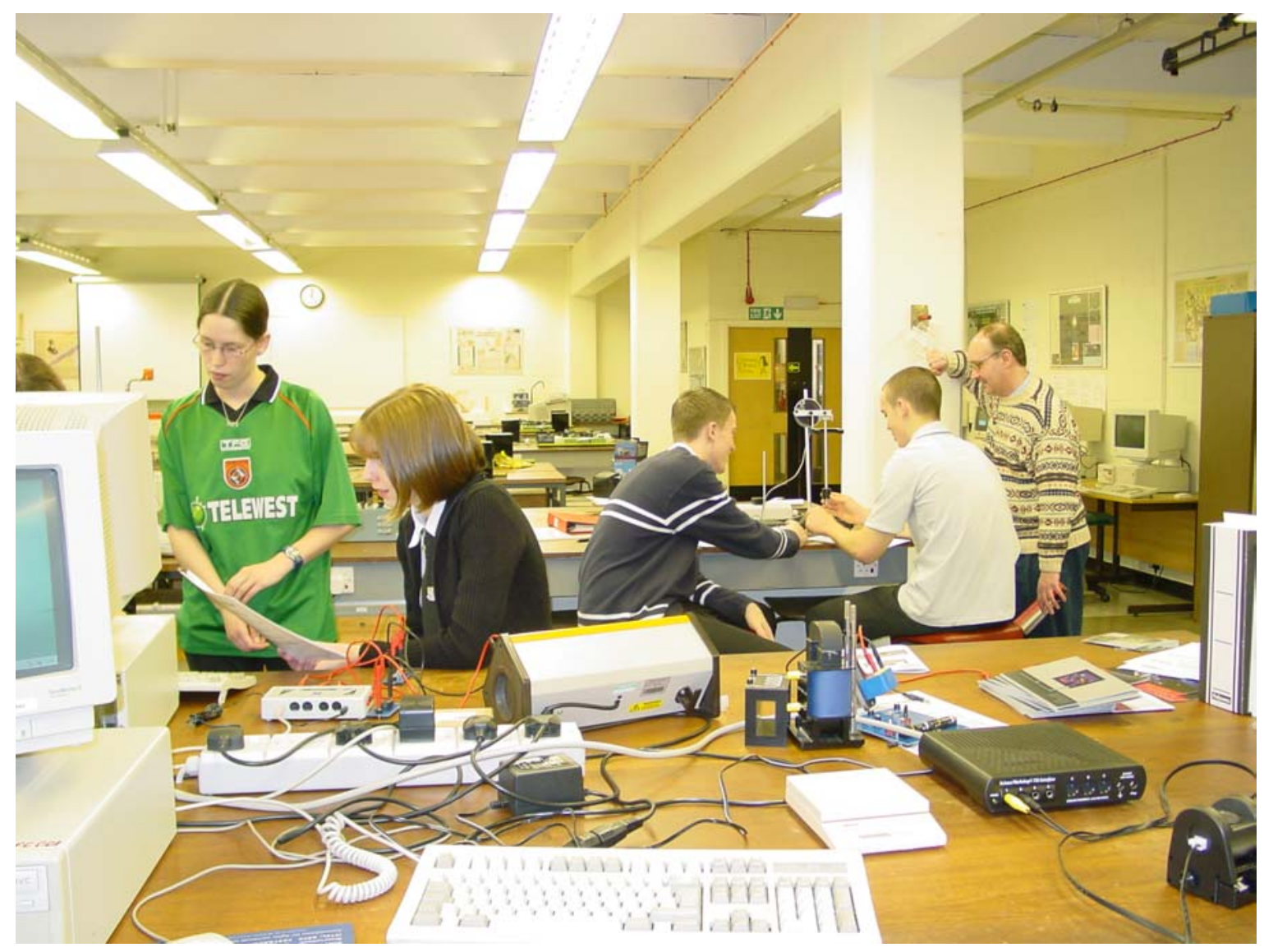

Figure 1 School and university students working together in the SCHOOLS Laboratory, with staff 
New Directions in the Teaching of Physical Sciences

\section{Author(s)}

Campbell White, Tynecastle High School, Edinburgh

Mike Steel, Department of Physics, Heriot-Watt University, Edinburgh

\section{Contact details}

Campbell White, Principal Teacher of Physics, Tynecastle High School, Edinburgh, seconded to Department of Physics, Heriot-Watt University

Mike Steel, Department of Physics, Heriot-Watt University, Edinburgh

Campbell White-C.White@hw.ac.uk

Mike Steel-m.r.steel@hw.ac.uk 